\title{
Optimal Placement of Distributed Generation for Reliability Benefit in Distribution Systems
}

\author{
N. Rugthaicharoencheep, A. Chalangsut \\ Department of Electrical Engineering, Faculty of Engineering, Rajamangala University of Technology Phra Nakhon, \\ Bangkok, Thailand \\ Email: nattachote.r@rmutp.ac.th
}

Received February, 2013

\begin{abstract}
A distributed generator is a small-scaled active generating unit located on or near the site where it is to be used. Several benefits have been realized by installing distributed generators in a distribution network. Among them is reliability improvement if their locations and sizes are appropriately determined. For this reason, reliability benefit is investigated in this paper with the main objective for the optimal placement and sizing of distributed generators in a distribution system to minimize the customer interruption cost subject to the maximum number of distributed generators, total capacity of distributed generators, bus voltage limits, current transfer capability of the feeders and only one distributed generator for one installation position. The technique employed to solve the minimization problem is based on a developed Tabu search algorithm and reliability worth analysis. The Tabu search algorithm is a local search that uses memory to avoid being trapped around a local neighborhood and help to move away from a local optimum solution. The reliability worth analysis provides an indirect measure for cost implication associated with power failure. The developed methodology is tested with a distribution system of Provincial Electricity Authority (PEA). Numerical results from the tests demonstrate that distributed generators can be used to promote the reliability of the distribution system.
\end{abstract}

Keywords: Distributed Generation; Reliability; Tabu Search; Distribution System

\section{Introduction}

Electricity has always been the major part of human development and it has gone through various changes with time. Traditionally, much of the electricity generated has been produced by large-scaled, centralized power plants using fossil fuels (e.g., coal, oil and gas), hydropower or nuclear power. The electrical energy is transmitted over long distances by extra high voltage (EHV) or ultra high voltage (UHV) transmission lines and from there the high voltage levels are converted to low voltage levels through distribution lines in the distribution system to end-use customers [1].

Such a centralized generation pattern, however, suffers a number of drawbacks, such as a high level of dependence on imported fuels that are very vulnerable, transmission losses, the necessity for continuous upgrading and replacement of the transmission and distribution facilities and therefore high operating cost, and environmental impact. In addition, as electric demand is substantially increasing as a result of economic and social growths, the construction of a large sized power plant is running into financial and technical difficulties, because it is capital intensive and needs considerable amount of time.
An ideal alternative on electric distributions to electric users is the installation of a small sized generator or commonly known as distributed generator (DG). DG is a small-scale active generating unit located on or near the site where it is to be used (i.e., in distribution systems). The primary energy resources of DG could be wind, solar, biomass, fuel cells and hydrogen, etc [2].

Although DGs have gained many positive effects, they still have some economic and technical issues to be addressed before their applications in the distribution system can be realized. The main objective of this paper is to investigate the impact of distributed generation on distribution system reliability. It is expected that reliability on the installation of DGs can be improved because they can be served as backup generation when a utility supply interruption occurs. In other words, some of the load points can still be electrically supplied by the DGs and therefore economic loss as a result of the power outage can be reduced. However, amount of reliability improvement depends on location and size of the DGs to be installed. It is therefore proposed in this paper a method to determine the optimal placement and sizing of DGs in a distribution system to minimize the customer interruption cost subject to system operational constraints [3]. 
The technique employed to solve the minimization problem is based on a developed Tabu search algorithm and reliabilty worth analysis. The Tabu algorithm systematically searches solutions expressed in forms of the location and size of DGs. The solution obtained will then be passed to reliability worth analysis to evaluate the quality of the solution. The process is repeated until the best solution has been found. The developed methodology is tested with a distribution system of Provincial Electricity Authority (PEA) with 26 load points.

\section{Tabu Search}

Tabu search is a meta-heuristic that guides a local heuristic search strategy to explore the solution space beyond local optimality [4]. The basic idea behind the search is a move from a current solution to its neighborhood by effectively utilizing a memory to provide an efficient search for optimality. The memory is called "Tabu list", which stores attributes of solutions. In the search process, the solutions are in the Tabu list cannot be a candidate of the next iteration. As a result, it helps inhibit choosing the same solution many times and avoid being trapped into cycling of the solutions [5]. The quality of a move in solution space is assessed by aspiration criteria that provide a mechanism for overriding the Tabu list. Aspiration criteria are analogous to a fitness function of the genetic algorithm and the Bolzman function in the simulated annealing.

In the search process, a move to the best solution in the neighborhood, although its quality is worse than the current solution, is allowed. This strategy helps escape from local optimal and explore wider in the search space. A Tabu list includes recently selected solutions that are forbidden to prevent cycling. If the move is present in the Tabu list, it is accepted only if it has a better aspiration level than the minimal level so far. Figure 1 shows the main concept of a search direction in Tabu search [6].

\section{Reliability Indices}

The basic distributed system reliability indices at a load point are average failure rate $\lambda$, average outage duration $r$, and annual outage duration $U$. With these three basic load point indices, the following system reliability indices can be calculated [7].

System average interruption frequency index (SAIFI)

$$
S A I F I=\sum \frac{\lambda_{i} N_{i}}{N_{i}}
$$

System average interruption duration index (SAIDI)

$$
S A I D I=\sum \frac{U_{i} N_{i}}{N_{i}}
$$

Customer average interruption duration index (CAIDI)

$$
C A I D I=\sum \frac{U_{i} N_{i}}{\lambda_{i} N_{i}}
$$

Average service availability index (ASAI)

$$
A S A I=\frac{\sum N_{i} \times 8760-\sum U_{i} N_{i}}{\sum N_{i} \times 8760}
$$

Average service unavailability index (ASUI)

$$
A S U I=1-A S A I=\frac{\sum U_{i} N_{i}}{\sum N_{i} \times 8760}
$$

Energy not supplied index (ENS)

$$
E N S=\sum L_{a(i)} U_{i}
$$

Average energy not supplied index (AENS)

$$
\text { AENS }=\frac{\sum L_{a(i)} U_{i}}{\sum N_{i}}
$$

where

$$
\begin{aligned}
\lambda_{i} & =\text { failure rate of load point } i \\
N_{i} & =\text { number of customers of load point } i \\
U_{i} & =\text { annual outage time of load point } i \\
L_{a(i)} & =\text { average load connected to load point } i \\
\lambda_{h} & =\text { failure rate of contingency } h \\
r_{h} & =\text { average outage time of contingency } h
\end{aligned}
$$

A basic approach to quantifying the worth of electric service reliability is to estimate customer interruption costs due to electric power supply interruptions. One convenient way is an interpretation of customer interruption costs in terms of customer damage functions. The customer damage functions can be determined for given customer types and aggregated to make sector customer damage functions (SCDF), which reflect economic consequences of supply interruption as a function of cost in different groups of customers [8].

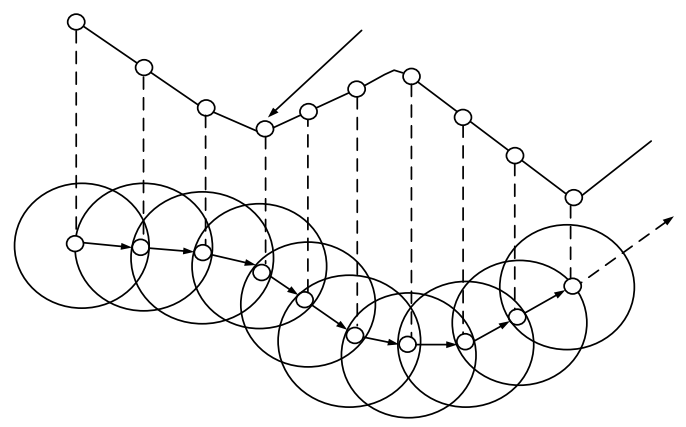

Figure 1. Search direction of Tabu search. 


\section{Problem Formulation}

\section{Objective function:}

$$
\text { Minimize ECOST }=\sum_{h=1}^{n_{h}} \sum_{i=1}^{n_{i}} L_{i} C_{h i}\left(r_{h}\right) \lambda_{h}
$$

\section{Constraints:}

Power flow equations:

$$
\begin{gathered}
P_{k}=\sum_{i=1}^{N_{B}}\left|Y_{i k} V_{i} V_{k}\right| \cos \left(\theta_{i k}+\delta_{k}-\delta_{i}\right) \\
Q_{k}=-\sum_{i=1}^{N_{B}}\left|Y_{i k} V_{i} V_{k}\right| \sin \left(\theta_{i k}+\delta_{k}-\delta_{i}\right)
\end{gathered}
$$

Voltage of each bus must be within specified limits:

$$
V_{k}^{\min } \leq V_{k} \leq V_{k}^{\max }
$$

Current transfer capability of feeders:

$$
I_{l} \leq I_{l}^{\max }, l \in\left\{1,2, \ldots N_{l}\right\}
$$

Maximum number of DGs to be installed:

$$
\sum_{k=1}^{N_{B}} e_{j k} \leq n_{D G} j \in\left\{1,2, \ldots N_{C}\right\}
$$

Maximum installed capacity of DGs:

$$
\sum_{k=1}^{N_{B}} \sum_{j=1}^{N_{C}} C_{j} \cdot e_{j k} \leq G
$$

Decision variables for the installation of a DG:

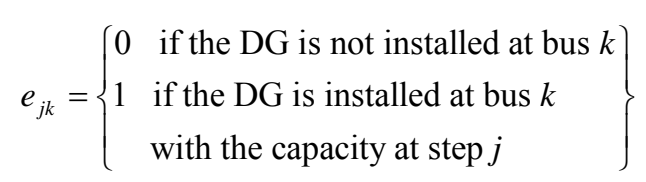

Only one DG can be installed at one position:

$$
\sum_{j=1}^{N_{C}} e_{j k} \leq 1 \quad k \in\left\{1,2, \ldots N_{B}\right\}
$$

where

$$
\begin{aligned}
& C_{h i} \quad=\begin{array}{l}
\text { Outage cost }(\$ / \mathrm{kW}) \text { of customer due to con- } \\
\text { tingency } h \text { with an outage duration of } r_{h}
\end{array} \\
& L_{h} \quad=\text { load at load point } i \\
& n_{i} \quad=\text { total number of load points } \\
& n_{h} \quad=\text { number of contingencies } \\
& P_{k} \quad=\text { power active power at bus } \\
& Q_{k} \quad=\text { power reactive power at bus } \\
& Y_{i k} \quad=\text { element }(i, k) \text { in bus admittance matrix } \\
& \theta_{i k} \quad=\text { angle of } Y_{i k} \\
& \delta_{k} \quad=\text { voltage angle at bus }
\end{aligned}
$$

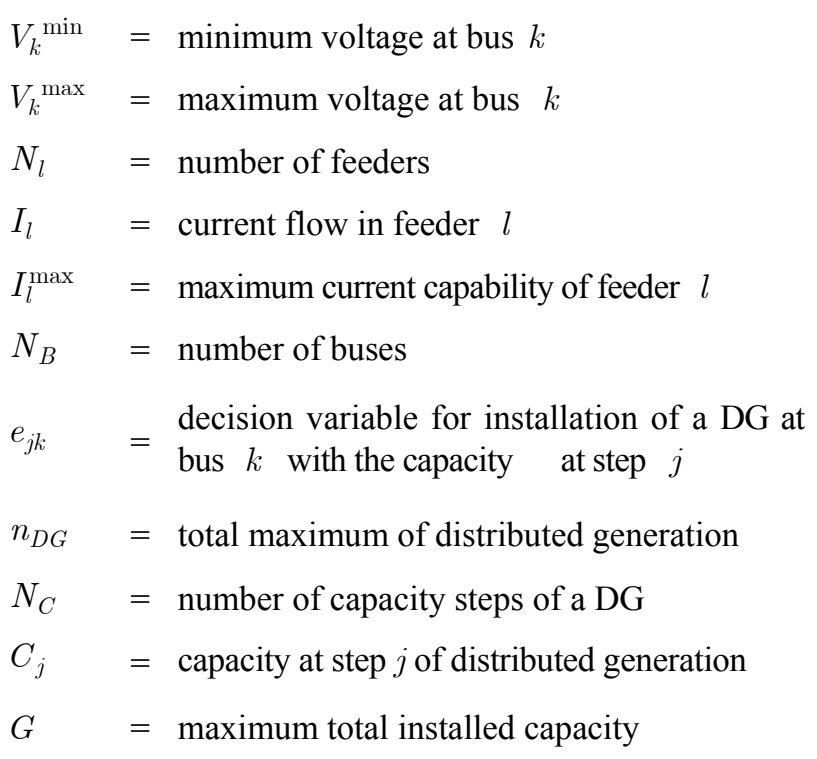

\section{Solution Algorithm}

The solution algorithm for the problem is described step by step as follows:

Step 1: Randomly select a feasible solution from the search space: $\mathrm{S}_{0} \in \Omega$. Set the size of a Tabu list, maximum iteration and iteration index $\mathrm{m}=1$.

Step 2: Let the initial solution obtained in step 1 be the current solution and the best solution: $\mathrm{S}_{\text {best }}=\mathrm{S}_{0}$, and $\mathrm{S}_{\text {current }}=\mathrm{S}_{0}$.

Step 3: Perform a power flow analysis to determine whether the current solution satisfies the constraints defined in (9) and (10). A penalty factor is applied for constraint violation.

Step 4: Calculate ECOST using (8) with consideration of load point restoration.

Step 5: Calculate the aspiration level of $S_{\text {best }}: f_{\text {best }}=$ $\mathrm{f}\left(\mathrm{S}_{\text {best }}\right)$. The aspiration level is the sum of ECOST and a penalty function.

Step 6: Generate a set of solutions in the neighborhood of $\mathrm{S}_{\text {current }}$. This set of solutions is designated as $\mathrm{S}_{\text {neighbor. }}$.

Step 7: Calculate the aspiration level for each member of $\mathrm{S}_{\text {neighbor }}$, and choose the one that has the highest aspiration level, $\mathrm{S}_{\text {neighbor_best }}$.

Step 8: Check whether the attribute of the solution obtained in step 7 is in the Tabu list. If yes, go to step 9 , or else $S_{\text {current }}=S_{\text {neighbor_best }}$ and go to step 10. 
Step 9: Accept $\mathrm{S}_{\text {neighbor best }}$ if has a better aspiration level than $\mathrm{f}_{\text {best }}$ and set $S_{\text {current }}=S_{\text {neighbor best }}$, or else select a next-best solution that is not in the Tabu list to become the current solution.

Step 10: Update the Tabu list and set $m=m+1$.

Step 11: Repeat steps 6 to 10 until the specified maximum iteration has been reached and report the best solution.

where

$$
\begin{array}{ll}
S_{0} & =\text { initial solution } \\
\Omega & =\text { search space } \\
S_{\text {best }} & =\text { best solution in search space } \\
S_{\text {current }} & =\text { current solution in search space } \\
f_{\text {best }} & =\text { objective function of } S_{\text {best }} \\
S_{\text {neighbor }} & =\text { neighborhood solutions of } S_{\text {current }} \\
S_{\text {neighbor_best }} & =\text { best solution of } S_{\text {neighbor }}
\end{array}
$$

\section{Case Study}

The developed Tabu search algorithm was tested with a distribution system of PEA consisting of two feeders KWA01 and KWA06. The system is modified [9] to include disconnecting switches and fuses so that the benefit of DGs can be realized. There are 6 load points in feeder KWA01 and 20 load points in feeder KWA06. The configuration of the system is shown in Figure 2. The maximum iteration for Tabu search is 1,000 . The minimum and maximum voltages for each bus are 0.95 p.u. and 1.05 p.u. The sizes of DGs are $100 \mathrm{~kW}, 200 \mathrm{~kW}, 300$ $\mathrm{kW}, 400 \mathrm{~kW}$ and $500 \mathrm{~kW}$. The failure of a transformer is recovered by repair. All the protective devices and DGs are assumed to be fully reliable. Seven cases are investigated in this case study.

Case 1: No DG is installed in the system.

Case 2: No more than one DG can be installed in the system.

Case 3: No more than two DGs can be installed in the system.

Case 4: No more than three DGs can be installed in the system.

Case 5: Total installed capacity of DGs cannot be greater than $600 \mathrm{~kW}$ and no more than four DGs can be installed in the system

Case 6: The same as case 5 except that total installed capacity of DGs cannot be greater than $800 \mathrm{~kW}$.

Case 7: The same as case 5 except that total installed capacity of DGs cannot be greater than $1,000 \mathrm{~kW}$

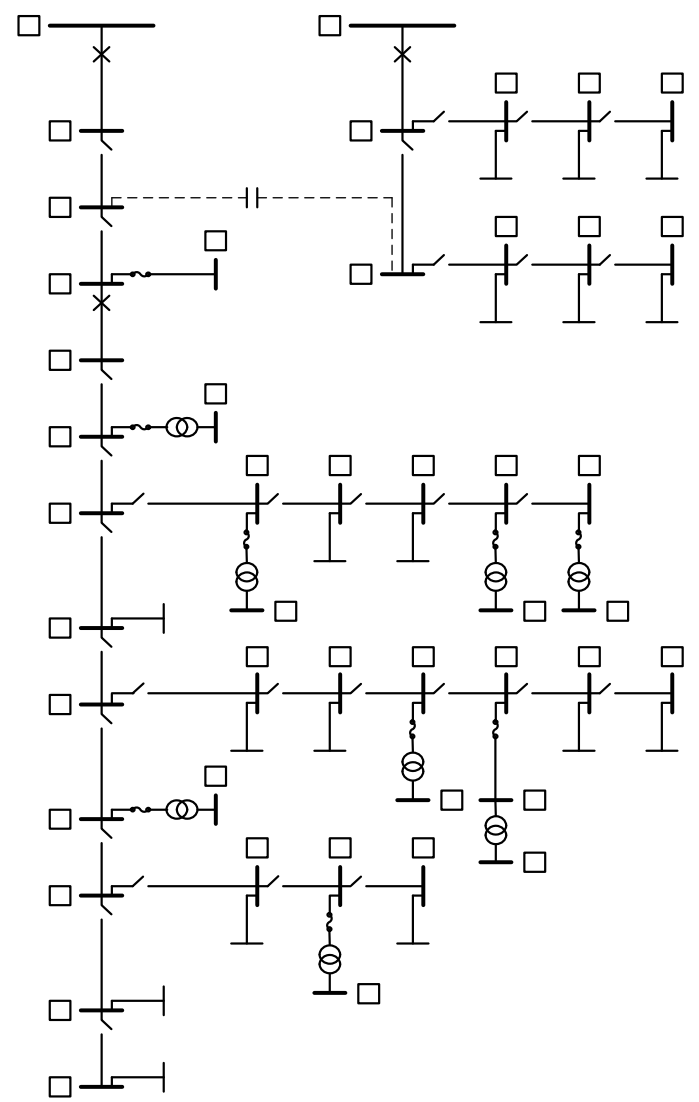

Figure 2. Single line diagram of two feeders of PEA.

The results from the case study are shown in Tables $\mathbf{1}$, 2 and 3. All the cases have the same SAIFI because this index depends only on the reliability of components (e.g., lines, transformers) and is not affected distributed generators to be installed.

We can see that the overall reliability indices of cases 2 to 7 are improved compared with that of case 1 (base case). In cases 2,3 , and 4 , where the number of DGs is limited at 1,2, and 3 respectively, see reductions in the system ECOST. It is very interesting to note that the constraint given in (13) is binding for these three cases.

Table 1. Location and capacity of distributed generators.

\begin{tabular}{ccccc}
\hline \multirow{2}{*}{ Case } & \multicolumn{2}{c}{ Location of DG (bus) } & \multicolumn{2}{c}{ Capacity of DG (kW) } \\
\cline { 2 - 5 } & KWA01 & KWA06 & KWA01 & KWA06 \\
\hline 1 & - & - & - & - \\
2 & - & 24 & - & 500 \\
3 & 6 & 24 & 300 & 500 \\
4 & 6,9 & 24 & 300,300 & 500 \\
5 & 7 & 24 & 100 & 500 \\
6 & 9 & 24 & 300 & 500 \\
7 & 9 & 18,24 & 300 & 200,500 \\
\hline
\end{tabular}


Table 2. Reliability indices of case study 1-4.

\begin{tabular}{ccccc}
\hline $\begin{array}{c}\text { Reliabil- } \\
\text { ity }\end{array}$ & \multicolumn{4}{c}{ Case } \\
\hline indices & 1 & 2 & 3 & 4 \\
\hline SAIFI & 7.33998 & 7.33998 & 7.33998 & 7.33998 \\
SAIDI & 17.8899 & 14.7669 & 14.7593 & 14.7484 \\
CAIDI & 2.43733 & 2.01184 & 2.01080 & 2.00932 \\
ASAI & 0.997958 & 0.998314 & 0.998316 & 0.998317 \\
ASUI & 0.002042 & 0.001686 & 0.001684 & 0.001683 \\
ENS & $45,746.8$ & $42,008.7$ & $41,347.6$ & $40,833.1$ \\
AENS & 116.404 & 106.892 & 105.210 & 103.007 \\
ECOST & $1,787,061$ & $1,622,746$ & $1,592,748$ & $1,569,397$ \\
\hline
\end{tabular}

Table 3. Reliability indices of case study 5-7.

\begin{tabular}{cccc}
\hline \multirow{2}{*}{$\begin{array}{c}\text { Reliability } \\
\text { indices }\end{array}$} & \multicolumn{3}{c}{ Case } \\
\cline { 2 - 4 } & 5 & 6 & 7 \\
\hline SAIFI & 7.33998 & 7.33998 & 7.33998 \\
SAIDI & 14.7633 & 14.7521 & 14.3976 \\
CAIDI & 2.011356 & 2.009821 & 1.961523 \\
ASAI & 0.998315 & 0.998315 & 0.998356 \\
ASUI & 0.001685 & 0.001684 & 0.001643 \\
ENS & $41,958.9$ & $41,494.1$ & $41,313.7$ \\
AENS & 106.766 & 105.583 & 105.124 \\
ECOST & $1,620,491$ & $1,599,395$ & $1,592,721$ \\
\hline
\end{tabular}

The reason is that to minimize the system ECOST, as many DGs as possible should be installed. However, for example, in case 3 , a $300 \mathrm{~kW}$ unit, instead of a $400 \mathrm{~kW}$ or a $500 \mathrm{~kW}$ unit, is placed at bus 6 . An explanation for this is that the $300 \mathrm{~kW}$ unit is sufficient for the demand at bus 6 . Had the 400 or $500 \mathrm{~kW}$ unit been placed at bus 6 the system ECOST would have been the same. Likewise, a $300 \mathrm{~kW}$ in case 4 installed at bus 9 can sufficiently cover the demands of LP4, LP5, and LP6.

With regard to cases 5,6 , and 7 , the constraint on total capacity of DGs is binding but the constraint on maximum number of DGs is not. The same reason given in cases 2, 3, and 4 are also used to explain the binding of these three cases. It is observed that a DG, if its size is large enough, tends to be installed at the end of a feeder. Such a placement is reasonable because the load point at the end of feeder has the highest failure rate and therefore most frequently needs a backup generation. In addition, the DG is able to supply power to upstream load points.

\section{Conclusions}

This paper has presented a Tabu search-based method for optimal placement of distributed generation in distribution systems with the main objective to maximize reliability benefits described in forms of the customer interruption cost. From reliability point of view, distributed generators are served as back up generation for load points that would otherwise have been left disconnected until the repair of a faulted component had been completed. The effectiveness of the proposed method was demonstrated by a case study of a distribution network of PEA with 26 load points. It can be seen from the case study that distributed generators can reduce the customer interruption cost and therefore improve the reliability of the system.

\section{REFERENCES}

[1] T. Wang, L. F. Ochoa and G. P. Harrison, "DG Impact on Investment Deferral: Network Planning and Security of Supply," IEEE Transaction Power Systems, Vol. 25, No. 2, 2010, pp. 1134-1141. doi:10.1109/TPWRS.2009.2036361

[2] J. Zhang, H. Cheng and C. Wang, "Technical and Economic Impacts of Active Management on Distribution Network," Electrical Power and Energy Systems, Vol. 31, No. 2-3, 2009, pp. 130-138. doi:10.1016/j.ijepes.2008.10.016

[3] J. Mutale, "Benefits of Active Management of distribution networks with distributed generation," in Proc. Power System Conf. and Exposition, 2006, pp. 601-606.

[4] D. Berna and A. Cigdem, "Simulation Optimization Using Tabu Search," Proceedings of the 2000 Winter Simulation Conference, 2000, pp. 805-810.

[5] F. Glover, Tabu Search-Part I. ORSA J. Computing, Vol. 1, No. 3, 1989.

[6] M. Hiroyuki and O. Yoshihiro, Parallel Tabu Search for Capacitor Placement in Radial Distribution System. Power Engineering Society Winter Meeting, 23-27 January, Vol. 4, 2000, pp. 2334-2339.

[7] R. Billinton and R. N. Allan, "Reliability Evaluation of Power Systems," Pitman Advanced Publishing Program, 1984. doi:10.1007/978-1-4615-7731-7

[8] L. Goel and R. Billinton, "A Procedure for Evaluating Interrupted Energy Assessment Rates in an Overall Electric Power System," IEEE Transaction on Power Systems, Vol. 6, No. 4, 1991, pp. 1398-1403. doi:10.1109/59.116981

[9] K. Kanokwan and S. Sirisumrannukul, Optimal Placement of Sectionalizing Switches in Redial Distribution System by a Genetic Algorithm, The $2^{\text {nd }}$ Greater Mekong Subregion Academic and Research Network (GMSARN) International Conference, Pattaya, Thailand 12-14 Nov., pp. 1-7. 2007. 


\section{Appendix}

Table A1. Customer data of feeder KWA01.

\begin{tabular}{ccccc}
\hline \multirow{2}{*}{$\begin{array}{c}\text { Load } \\
\text { Point }\end{array}$} & $\begin{array}{c}\text { Number of } \\
\text { Customer }\end{array}$ & Type & \multicolumn{2}{c}{ Demand } \\
\cline { 4 - 5 } & & & P (kW) Average & Q (kVAR) \\
\hline LP1 & 1 & Large Business & 700 & 433.83 \\
LP2 & 1 & Large Business & 700 & 433.83 \\
LP3 & 1 & Medium Business & 220.5 & 136.65 \\
LP4 & 1 & Medium Business & 35 & 21.69 \\
LP5 & 1 & Medium Business & 105 & 65.07 \\
LP6 & 1 & Medium Business & 105 & 65.07 \\
\hline
\end{tabular}

Table A2. Customer data of feeder KWA06.

\begin{tabular}{|c|c|c|c|c|}
\hline \multirow{2}{*}{$\begin{array}{l}\text { Load } \\
\text { Point }\end{array}$} & \multirow{2}{*}{$\begin{array}{l}\text { Number of } \\
\text { Customer }\end{array}$} & \multirow[b]{2}{*}{ Type } & \multicolumn{2}{|c|}{ Demand } \\
\hline & & & $\begin{array}{l}\mathrm{P}(\mathrm{kW}) \\
\text { Average }\end{array}$ & Q (kVAR) \\
\hline LP1 & 1 & Large Business & $3,130.75$ & 1,940 \\
\hline LP2 & 105 & Residence & 32.50 & 20.14 \\
\hline LP3 & 31 & Residence & 9.75 & 6.04 \\
\hline LP4 & 1 & Medium Business & 110.25 & 68.33 \\
\hline LP5 & 31 & Residence & 9.75 & 6.04 \\
\hline LP6 & 31 & Residence & 9.75 & 6.04 \\
\hline LP7 & 21 & Residence & 6.50 & 4.03 \\
\hline LP8 & 1 & Government & 45.50 & 28.20 \\
\hline LP9 & 21 & Residence & 6.50 & 4.03 \\
\hline LP10 & 1 & Small Business & 10.50 & 6.51 \\
\hline LP11 & 1 & Medium Business & 175 & 108.46 \\
\hline LP12 & 31 & Residence & 9.75 & 6.04 \\
\hline LP13 & 84 & Residence & 26 & 16.11 \\
\hline LP14 & 1 & Medium Business & 56 & 34.71 \\
\hline LP15 & 1 & Medium Business & 175 & 108.46 \\
\hline LP16 & 1 & Government & 22.75 & 14.10 \\
\hline LP17 & 1 & Government & 17.50 & 10.85 \\
\hline LP18 & 1 & Government & 35 & 21.69 \\
\hline LP19 & 21 & Residence & 6.50 & 4.03 \\
\hline LP20 & 1 & Government & 9.75 & 6.04 \\
\hline
\end{tabular}

Table A3. Customer damage function.

\begin{tabular}{ccccc}
\hline \multirow{2}{*}{ Type } & \multicolumn{4}{c}{$\begin{array}{c}\text { Duration in Hours and Interruption Cost } \\
(\text { Baht/kW) }\end{array}$} \\
\cline { 2 - 5 } & $1 \mathrm{hr}$ & $2 \mathrm{hr}$ & $4 \mathrm{hr}$ & $8 \mathrm{hr}$ \\
\hline Residence & 8.694 & 19.050 & 39.762 & 80.716 \\
Small Business & 166.172 & 288.467 & 591.748 & $1,054.216$ \\
Medium Business & 55.006 & 92.647 & 193.661 & 363.221 \\
Large Business & 50.877 & 79.913 & 145.614 & 251.938 \\
Government & 20.025 & 28.827 & 40.175 & 50.941 \\
\hline
\end{tabular}

Table A4. Reliability parameters of feeders KWA01 and KWA06.

\begin{tabular}{cccc}
\hline Component & $\lambda(\mathrm{f} / \mathrm{yr})$ & $r(\mathrm{hr})$ & $s w(\mathrm{hr})$ \\
\hline Transformers & 0.0150 & 200 & - \\
Line & 0.3700 & 5 & 1.06 \\
\hline
\end{tabular}

where $\lambda=$ failure rate of component; $r=$ repair time; $s w=$ switching time

Table A5. Type and length of feeder KWA01.

\begin{tabular}{ccc}
\hline Line No. & Type & Length $(\mathrm{km})$ \\
\hline 1 & SAC 185 & 1.0760 \\
2 & PIC 185 & 0.9740 \\
3 & PIC 185 & 0.0066 \\
4 & PIC 185 & 0.1960 \\
5 & SAC 185 & 2.1750 \\
6 & SAC 185 & 0.4150 \\
7 & SAC 185 & 0.0610 \\
8 & SAC 185 & 0.0130 \\
9 & SAC 185 & 0.9800 \\
\hline
\end{tabular}

Table A6. Type and length of feeder KWA06.

\begin{tabular}{|c|c|c|}
\hline Line No. & Type & Length $(\mathrm{km})$ \\
\hline 1 & SAC 185 & 8.7400 \\
\hline 2 & SAC 185 & 0.3830 \\
\hline 3 & SAC 185 & 0.4290 \\
\hline 4 & SAC 185 & 0.2890 \\
\hline 5 & SAC 185 & 3.0060 \\
\hline 6 & ACSR 50 & 0.1900 \\
\hline 7 & ACSR 50 & 1.0690 \\
\hline 8 & ACSR 50 & 0.8540 \\
\hline 9 & ACSR 50 & 0.0170 \\
\hline 10 & ACSR 50 & 0.2220 \\
\hline 11 & ACSR 50 & 0.1580 \\
\hline 12 & ACSR 50 & 0.0810 \\
\hline 13 & ACSR 50 & 0.5080 \\
\hline 14 & ACSR 50 & 0.0640 \\
\hline 15 & ACSR 50 & 0.3120 \\
\hline 16 & ACSR 50 & 0.0510 \\
\hline 17 & ACSR 50 & 0.4660 \\
\hline 18 & ACSR 50 & 0.0910 \\
\hline 19 & ACSR 50 & 0.4100 \\
\hline 20 & ACSR 50 & 0.1660 \\
\hline 21 & ACSR 50 & 0.3190 \\
\hline 22 & ACSR 50 & 0.5050 \\
\hline 23 & ACSR 50 & 0.1300 \\
\hline 24 & ACSR 50 & 0.3940 \\
\hline 25 & ACSR 50 & 0.6930 \\
\hline 26 & ACSR 50 & 0.4300 \\
\hline 27 & ACSR 50 & 0.2910 \\
\hline 28 & ACSR 50 & 0.0910 \\
\hline
\end{tabular}

